\title{
Evaluating Chlorophyll-a Changes During Algal Bloom in Three Gorges Reservoir Using an Extended WASP Model
}

\author{
Li Jian ${ }^{1,2}$, Qin Xiaosheng ${ }^{2, a}$ and Chen Ming ${ }^{2}$ \\ ${ }^{1}$ Yangtze River Scientific Research Institute, No.23 Huangpu Road, Wuhan, China \\ ${ }^{2}$ School of Civil and Environmental Engineering, Nanyang Technological University, 50 Nanyang Ave., Singapore
}

\begin{abstract}
Algal bloom in Three Gorges Reservoir (TGR) and one of its tributaries, Xiangxi River (XR), have become major concerns and the dynamic changes of such events were investigated using the hydrodynamic model SELFE and the extended Water Quality Analysis Simulation Program (WASP) model to address nutrient and biomass dynamics. The model has taken into consideration the surface wind, heat fluxes, oxygen exchanges, solar radiations and boundary conditions from main river channel and tributaries. As an extension to our previous work, this study aimed to report in more detailed the result of chlorophyll- $a$ simulations, where the field observed data of algal blooms in TGR in 2007 was used for calibration and the horizontal and vertical distributions of phytoplankton biomass (based on chlorophyll-a) were presented. It was found that the chlorophyll-a concentration characterized as algal biomass was influenced by many complex factors. Further study results are yet to be reported.
\end{abstract}

\section{Introduction}

The Three Gorges Reservoir (TGR), with a storage capacity around 39.3 billion $\mathrm{m}^{3}$, is located in the upstream of the Yangtze River, China. The Yangtze River and its tributaries in the reservoir area are featured by both narrow and deep boundaries. Meanwhile, obvious exchanges of water body and various solutes (including nutrients and suspended sediments) occur between the mainstream and the tributaries under frequent reservoir operations (for the purpose of flood control and hydroelectricity generation). Various pollutants flow into the reservoir especially the tributary of Xiangxi River (XR) from many chemical factories and sanitary sewage systems, which caused frequent the algal bloom events in the tributaries after water impoundment in 2003.

Over the past years, many researchers have conducted field observations and numerical simulations for studying problems of water quality deterioration and ecosystem evolution in TGR and its tributaries. Particularly, many hydrodynamic and water quality models have been applied for examining the TGR algal blooms, which include 1-Dimensional [1], vertical 2-Dimensional [2] and 3-Dimensional [3] models. From the previous works, it was found that the flowing field, solute transport and phytoplankton biomass evolution in TGR present significant spatial and temporal variations [4]; hence, a 3-dimensional model is considered better suited for the modeling purpose. Generally, the 3-dimensioal models can be divided into structured and unstructured meshes

\footnotetext{
${ }^{a}$ Corresponding author: xsqin@ntu.edu.sg
} 
considering the topographical structure. For example, Dai et al. (2013) used a structured-mesh model, Delft3D, for modeling the TGR water quality [3]; however, the model cannot well follow the complex boundary and subaqueous relief in TGR. Therefore, in this study, an unstructured mesh water quality model was used based on the hydrodynamic model SELFE [5] and the Water Quality Analysis Simulation Program (WASP) model [6]. The detailed structure and general performance of this model was initially reported in $\mathrm{Li}$ [7]. The WASP model used here has been extended considering the updated research literatures [8] and the local characteristics about TGR water quality problem [4]. As an continual work to the previous study [7], the algal bloom occurred in 2007 was more extensively simulated and reported by the coupled models aiming at understanding the changing process of the horizontal and vertical distribution of phytoplankton biomass (chlorophyll-a).

\section{Methodology}

The coupled model was based on SELFE [5] and the extend WASP model [6] considering the most updated water quality research works [8]. The coupled hydrodynamic and extended WASP model covers the essential physiochemical and biological processes in the aquatic ecosystem in TGR in addition to the effect of sediments including suspended and bottom layer sediment on the water quality dynamics. The model also considers the interactions among many factors like hydrodynamics, water temperature, underwater light intensity, nutrients, dissolved oxygen (DO), biochemical oxygen demand (BOD) and phytoplankton biomass (denoted by chlorophyll- $a$ concentration). The author has added more modules into the original WASP model including nonlinear adsorption-desorption interaction between suspended sediment and phosphate, solutes exchange between bottom sediment layer and overlying water considering bottom stress [7-9]. Meanwhile, the Nitrogen and Phosphorus nutrients as Total Nitrogen (TN) and Total Phosphorus (TP) used in the model have been divided into organic and inorganic nutrients including nitrate $\left(\mathrm{NO}_{3}\right)$, ammonia $\left(\mathrm{NH}_{4}\right)$, organic nitrogen $(\mathrm{ON})$, phosphate $\left(\mathrm{PO}_{4}\right)$ and organic phosphorous $(\mathrm{OP})[9,10]$. For technical details, readers are referred to literatures [6-10].

\section{Study Case}

The XR (with a length about $94 \mathrm{~km}$, flowing through Xingshan and Zigui) is situated in the Hubei Province of China. Many other tributaries exist in TGR along the Yangtze River including Duanfangxi, Tongzhuanghe, and Qingshuihe, Yuanshuihe [7]. Since the discharge and pollutant load into XR are predominantly larger [11], other tributaries were considered as closed bay in the simulation. Serious water quality problems had occurred in these tributaries due to insufficient water mixing or flowing based on the in-situ observation [4]. Therefore, a high initial concentration of nutrients and chlorophyll- $a$ is used in the calculation. The hydrodynamic condition at local tributaries is closely linked to the Yangtze River's major channel flow. For instance, there existed backflow mixing and vertical water exchange for the period of water impoundment and water discharging processes of TGR. Hence, the physiochemical and biological parameters close to the XR outlet would act together with the main river flow. Therefore, the water body exchange between Yangtze River and the tributaries influencing the algal bloom process was also simulated.

The computational domain including Yangtze River, Xiangxi River, Gaolan River (the second-class tributary of Yangtze River) and some other first-class tributaries in TGR is discretized with 15,282 horizontal triangle elements [7]. A total of 11 observation stations in XR and 6 observation stations in Yangtze River were established along the river channel to measure parameters like nitrogen, phosphate, suspended sediment, DO, BOD and chlorophyll- $a$ in surface water; in addition, the biochemical and hydrological data during algal blooms occurring in 2007 were also collected. In numerical modeling, the input boundary of the Yangtze River, XR and Gaolan River were defined following the hydrologic stations data (i.e. discharge) during the events of actual algal blooms; the TGR dam boundary condition (i.e. water levels) was based on the actual reservoir operation data. 


\section{Results}

The SELFE-WASP model was calibrated using the measured data in 2007 when the algal blooms, hydrodynamics, and water quality were observed together and the data were suitable for the calibration of the 3D coupled modeling. The model calibration efforts for flow velocity were based on implementation of SELFE modeling. The main parameters of WASP were estimated many times in order to generate a reasonable fit of the observed data for algal bloom process. Other model parameters were selected based on the works from the previous publications [1-3]. The estimated or selected parameter values were found within the intervals reported by Justic and Wang [8]. And the calibrated parameters used in modeling (not listed here) can reproduce the algal bloom process (chlorophyll-a) well. One example in Gaoyang Station is shown in Fig. 1, where the simulated chlorophyll- $a$ concentration agreed well with the observed data at the 5-m depth under the water surface. Note the simulated and observed vertical profiles of chlorophyll- $a$ concentration at Gaoyang Station was not shown here.

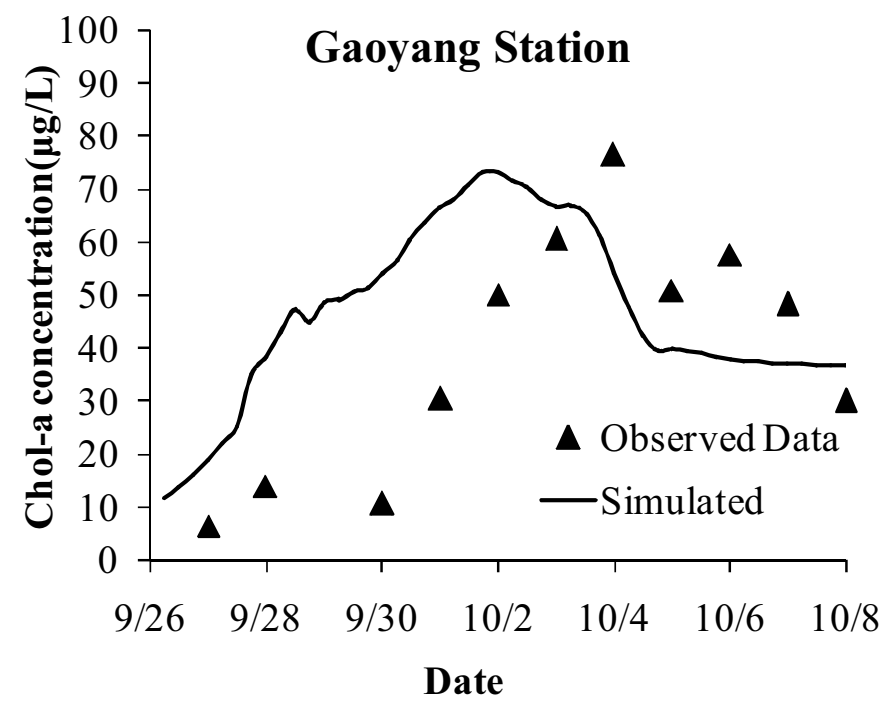

Figure 1. Observed vs. simulated concentrations in calibration of chlorophyll- $a$.

Figure 2 presents the results of horizontal distributions of flowing velocity and chlorophyll- $a$ concentration. The flowing velocity in Yangtze River was obviously higher (the maximum velocity can reach up to $0.3 \mathrm{~m} / \mathrm{s}$ ) than that in the Xiangxi tributary (the velocity was around $0 \mathrm{~m} / \mathrm{s}$ ) as shown in Figure 2(a) which would lead to different algal reproduction phenomenon, the backflow happened in tributary Xiangxi River would also decrease the nutrients and phytoplankton transportations into the Yangtze River. Meanwhile, the vortex at the confluence could also be seen in Figure 2(a) which was caused by different flowing directions from Yangtze River and Xiangxi River. The chlorophyll- $a$ concentration characterized as phytoplankton biomass was preliminarily simulated considering many factors influencing the growth, death and settling down of phytoplankton cells. The horizontal distribution of chlorophyll- $a$ concentration at both water surface and bottom can provide the information about the algal bloom locations in the TGR area in Figure 2(b). Obviously, the algal bloom event mainly occurs in the upper reach of XR from Pingyikou to the confluence of XR and Gaolan River (Xiakou) [7]. Meanwhile, the bottom Chlorophyll-a concentration was also obviously high at the shallow water zone like Pingyikou and Tongzhuanghe [7]. The phytoplankton biomass in the Yangtze River was relatively lower than that in the tributaries. Generally, the coupled model could provide reasonable reproduction of the algal bloom event, and the event occurring locations could be preliminarily analyzed through the proposed modeling study. 
(a)
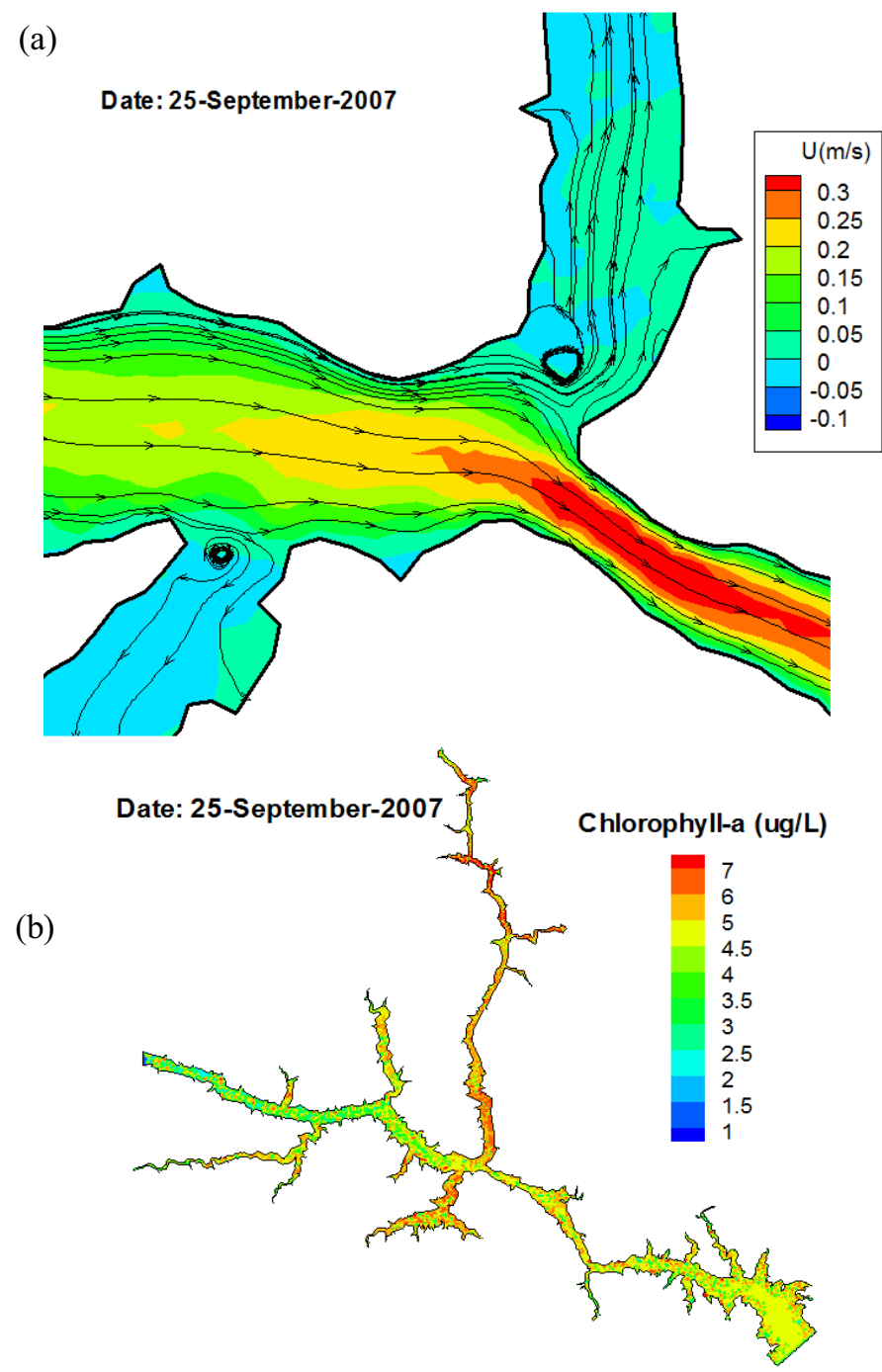

Figure 2. Horizontal distribution of variables (a) Flowing velocity at the confluence of Xiangxi River and Yangtze River and (b) Chlorophyll-a concentration near riverbed.

Figure 3 shows the results of vertical distribution of chlorophyll-a concentration. The phytoplankton cells at water surface can receive more lighting for photosynthesis than that in the deeper water column, which would lead to obvious differences of vertical chlorophyll- $a$ concentration. Because of over-saturated phenomenon caused by solar irradiation that will inhibit the photosynthesis, the chlorophyll- $a$ concentration beneath the water surface around water depth of $5.0 \sim 20.0 \mathrm{~m}$ reaches the maximum value. The chlorophyll- $a$ concentration is not the highest at the water surface as shown in Figure 3. The vertical concentration profiles of chlorophyll-a show stratifications in both the XR tributary (Fig. 3a) and the Yangtze River mainstream (Figure 3b). The chlorophyll- $a$ concentration in XR tributary was obviously higher than that in Yangtze River mainstream because the relatively high flowing speed could increase the self-cleaning capability and also more physical transport would decrease the phytoplankton biomass in Yangtze River mainstream as shown in Figure 3(a). The relatively stable flow in XR provides a suitable condition for the algal reproduction that has led to locally high chlorophyll- $a$ concentrations; while the chlorophyll- $a$ concentration in the Yangtze River is more related to the inflow boundary condition. 

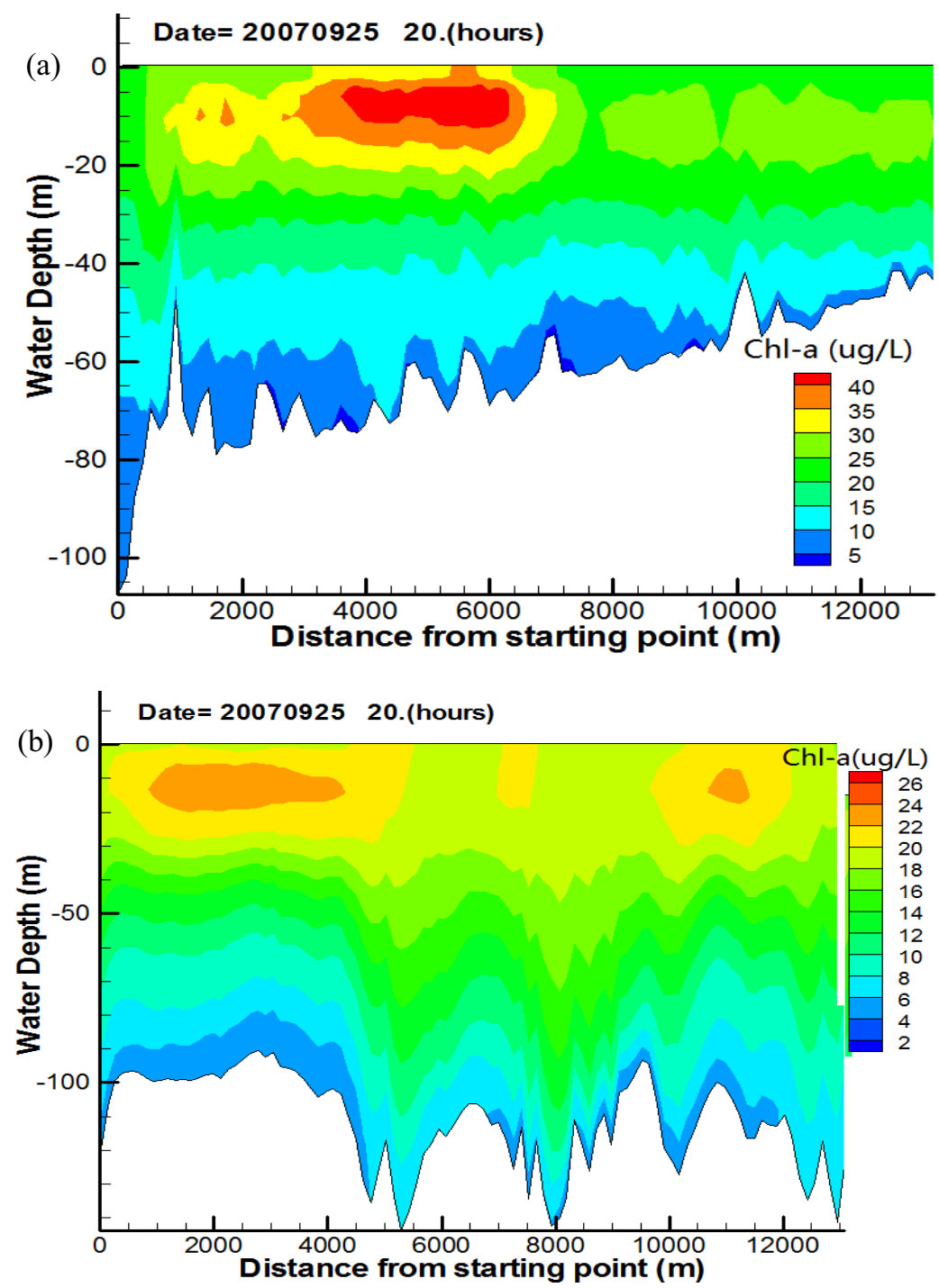

Figure 3. Vertical concentration profile of chlorophyll- $a$ at: (a) XR and (b) Yangtze River.

\section{Conclusion}

This study presents a preliminary numerical simulation on the spatial-temporal variation of chlorophyll- $a$ by using a numerical model coupling SELFE and an extended WASP module. The model was demonstrated effective for the case study of TGR and XR algal bloom event in 2007 [7]. The simulation domain covered Yangtze River and many tributaries which was different from the only simulation about XR algal bloom in the author's previous works [8, 9]. Reasonable tendencies of chlorophyll- $a$ changes and its concentration were preliminarily examined from the numerical modeling. The algal bloom occurring locations could be analyzed successfully through the proposed model. Further studies on other water quality constituents are yet to be reported more thoroughly in the near future. 


\section{Acknowledgement}

This material is based on research/work supported by the National Science Foundation of China (51309021) and in part by the Land and Liveability National Innovation Challenge under L2 NIC Award No. L2NICCFP1-2013-3 (WBS no.: M4061545.D63). We are also grateful to the Xiangxi River Ecological Observation Station of China, Three Gorges University for providing the field observation data.

\section{References}

[1] L. Wang, Z. Yu and H. Dai, Water Science and Engineering, 2, 16-24, (2009).

[2] J. Lian, Y. Yao and C. Ma, Water, 6, 3200-3223, (2014).

[3] H. Dai, J. Mao and D. Jiang, PLOS ONE, 8, 1-14, (2013).

[4] T. Zheng, J. Mao and H. Dai, Science China Technological Sciences, 54, 1588-1598, (2011).

[5] Y. Zhang and M. B. António, Ocean Modelling, 21, 71-96, (2008).

[6] T.M. Wool, Water Quality Analysis Simulation Program (WASP) Version 6 User's Manual, US Environmental Protection Agency, Atlanta, (2001).

[7] J. Li, E-proceedings of the $36^{\text {th }}$ IAHR World Congress, The Hague, Netherlands, (2015).

[8] D. Justić and L. Wang, Continental Shelf Research, 72, 163-179, (2014).

[9] J. Li, D. Li and X. Wang, Journal of Environmental Sciences, 24, 1569-1578, (2012).

[10] J. Li, Z. Jin and W. Yang, Ecological Informatics, 22, 23-35, (2014).

[11]K. Zhu, Doctoral dissertation of Chinese Academy of Sciences, (2013). 